\title{
COMPOUND DECISION IN THE PRESENCE OF PROXIES
}

\author{
Noam Cohen, Eitan Greenshtein and Ya'acov Ritov \\ Israeli CBS and The Hebrew University
}

\begin{abstract}
We study the problem of incorporating covariates in a compound decision setup. It is desired to estimate the means of $n$ response variables, which are independent and normally distributed, and each is accompanied by a vector of covariates. We suggest a method that involves non-parametric empirical Bayes techniques and may be viewed as a generalization of the celebrated Fay-Herriot (1979) method. Some optimality properties of our method are proved. We also compare it numerically with Fay-Herriot and other methods, in a real data situation, where the goal is to estimate certain proportions in many small areas (Statistical-Areas). We also demonstrate our approach through the baseball data set, originally analayzed by $\operatorname{Brown}(2010)$.
\end{abstract}

Key words and phrases: compound decision, empirical Bayes

\section{Introduction}

The main purpose of this paper is to study and demonstrate how to incorporate compound decision techniques (CD), or almost equivalently, empirical Bayes (EB) methods, in the presence of explanatory variables. The ideas of CD/EB were developed in the 1950's by Robbins (1951, 1955, 1964), see the review papers by Copas (1969) and Zhang (2003). Compound decision (or Empirical Bayes) procedures, were shown to produce very efficient estimators in the simple setup where we have independent observations, $Y_{1}, \ldots, Y_{n}, Y_{i} \sim F_{\mu_{i}}$, and it is desired to estimate $\mu_{i}$, $i=1, \ldots, n$. A major case, on which we will concentrate, is when $F_{\mu_{i}}=N\left(\mu_{i}, 1\right)$.

We will focus on two types of EB procedures. One type is Parametric Empirical Bayes (PEB) procedure, where $\mu_{i}, i=1, \ldots, n$ are assumed to be realizations of independent random variables $M_{i}, i=1, \ldots, n, M_{i} \sim G, G=N\left(0, \tau^{2}\right)$, where $\tau^{2}$ is unknown and should be estimated from the data. When $n$ is large, the corresponding estimator (note, the exact variant of the corresponding estimator, depends on the method of estimating $\tau^{2}$ ), resembles the JamesStein estimator, see e.g., Efron and Morris (1973). The other type is the Non-Parametric Empirical Bayes (NPEB) procedure, where the above distribution $G$ is a member of a large non parametric family $\mathcal{G}$ of distributions. Two recent NPEB methods and approaches are Brown and Greenshtein (2009) and Jiang and Zhang (2009). 
The advantage of EB procedures, relative to more elementary (e.g., mle) procedures, occures as $n$ grows, and may become very significant in high dimensional problems when $n$ is large (e.g., $n \geq 3$ is needed for "Stein's paradox" to hold). A special advantage of NPEB procedures is expected in situations where the vector $\boldsymbol{\mu}=\left(\mu_{1}, \ldots, \mu_{n}\right)^{\prime}$ is sparse, see e.g., Greenshtein, Park and Ritov (2008), Brown and Greenshtein (2009).

Since modern statistical problems often involve high dimensional and sparse estimation problems, EB techniques should be embraced for such purposes, see, e.g., Efron (2003). However, apart from literature in small area estimation, e.g., Rao (2003), which follows the seminal paper of Fay and Herriot (1979), EB is hardly used in modern data analysis. A recent additional approach, which is very much related to ours is Jiang and Zhang (2010). We became aware of this later approach after completing most of this paper, we will elaborate on it in the sequel. One important assumption in most theoretical discussions of EB is that of the exchangeability of the sample. This assumption does not hold in most applied problems. Typically, each observation $Y_{i}$ is accompanied by some explanatory variables $X_{i 1}, \ldots, X_{i p}$, thus the observations are not exchangeable any more and simple symmetric decision procedures have no appeal. We elaborate in the following.

In our motivating example $Y_{i} \sim B\left(m_{i}, p_{i}\right)$, the binomial distribution, and we need to estimate $p_{1}, \ldots, p_{n}$, certain proportions, in $n$ (small) areas. The values of $p_{1}, \ldots, p_{n}$ are unknown constants to be estimated. In addition to the sample $Y_{1}, \ldots, Y_{n}$, we have a set of variables $\mathbf{X}_{1}, \ldots, \mathbf{X}_{\mathbf{n}}$ (fixed or random, but independent of $Y_{1}, \ldots, Y_{n}$ ) and hopefully $\mathbf{X}_{\mathbf{i}}$ can serve as proxies to $p_{i}, i=1, \ldots, n$. For example one dimensional covariates $X_{i} \sim B\left(k_{i}, \tilde{p}_{i}\right)$ where $\tilde{p}_{i}$ are "typically" close to $p_{i}$; alternatively $\boldsymbol{X}_{i}$ may be a vector of known parameters of area $i$, that might be "relevant" to the parameter of interest $p_{i}$, for example the socio-economic level of the region, its size, or mean age. We emphasize two elements. First, because of the proxies, $Y_{1}, \ldots, Y_{n}$ cannot be considered as "permutation invariants" or "exchangeable". Second, we do not believe that the observations follow standard regression models. The covariates are considered as proxies to $p_{i}$, but they are statistically independent of the $Y$ 's (whose only stochastic aspect comes from the binomial sampling), and may be only a rough approximation to $p_{1}, \ldots, p_{n}$.

Simple symmetric and permutation invariant procedures. In cases of total ignorance regarding the parameters of the variables in relation to their identity, e.g., a situation where $Y_{i} \sim N\left(\mu_{i}, 1\right)$ and there is an exchangeable multivariate prior on $\left(\mu_{1}, \ldots, \mu_{n}\right)$, procedures which are permutation invariant have a special appeal. Permutation invariant procedures $\Delta$ are such that for every permutation $\pi$,

$$
\Delta\left(Y_{1}, \ldots, Y_{n}\right)=\left(a_{1}, \ldots, a_{n}\right) \Longleftrightarrow \Delta\left(Y_{\pi(1)}, \ldots, Y_{\pi(n)}\right)=\left(a_{\pi(1)}, \ldots, a_{\pi(n)}\right) ;
$$

here $a_{i} \in A$, where $A$ is the ( abstract) action space. A simple class of exchangeable priors is where $\mu_{i}$ are realizations of i.i.d $M_{i} \sim G, i=1, \ldots, n$. The optimal procedures then belong to the class of 'simple symmetric decision functions', i.e., procedures $\Delta$ which are of the form:

$$
\Delta\left(Y_{1}, \ldots, Y_{n}\right)=\left(\delta\left(Y_{1}\right), \ldots, \delta\left(Y_{n}\right)\right)
$$


for a given $\delta$. For natural losses, given $G$, the optimal $\delta$ corresponds to the one dimensional Bayes procedure. On the relation and asymptotic equivalence between the above two classes, see Greenshtein and Ritov (2009). Given a loss function, consider an 'oracle' who knows the values of $\mu_{1}, \ldots, \mu_{n}$, but is required to use a permutation invariant procedure. EB and CD procedures may be viewed as an attempt to immitate the (unknown) procedure that an oracle would use. This is a very natural goal under 'total ignorance' or 'exchangeability'.

The appeal in using permutation invariant procedures and consequently EB procedures, is lost when exchangeability is lost, as in cases where there are explanatory variables. Assume $n=n_{1}+n_{2}$ and it is known that the first $n_{1}$ observations (say, hormone measurements), were taken from men, while the last $n_{2}$ were taken from women. Applying a permutation invariant procedure is equivalent to ignoring this potentially important information/explanatory-variable. However not all is lost, one may still apply EB procedure seperately on the first $n_{1}$ observations and on the last $n_{2}$ observations. The idea is that after accounting for the explanatory variable in this trivial manner, we arrive into (two groups of) exchangeable variables and applying EB procedures seperately on each group becomes appealing. In a similar manner, we will account for the information in the explanatory variables and then, after the information from the explanatory variables is accounted for and the "accounted observations" are closer to being exchangeable, we apply an EB procedure.

$\mathrm{EB}$ and $\mathrm{CD}$ are closely related notions and approaches. Under an EB formulation the parameters $\mu_{i}, i=1, \ldots, n$ are independent realizations from an unknown distribution $G$ and the aim is to approximate the corresponding Bayes rule; under a CD formulation the aim is to approximate the best decision rule within a class of procedures (e.g., simple-symmetric, permutation invariant), for the given $\boldsymbol{\mu}=\left(\mu_{1}, \ldots, \mu_{n}\right)^{\prime}$. In this paper we emphasize the CD approach. However, we will often use the more familiar EB notion, motivation and terminology.

Applying a (variant of) PEB method after accounting for the covariates, is in the spirit of the paper of Fay and Herriot, as shown in sub-section 2.2; it is currently the most common practice. Another approach for inference in the presence of explanatory variables is that of Lindley and Smith (1972), it is a parametric empirical Bayes approach, though different than that of Fay and Herriot.

In Section 2, we will suggest how EB could naturally be incorporated in problems with explanatory variables. We extend the Fay-Herriot approach and present its PEB and NPEB versions. We show asymptotic optimality of NPEB.

In section 3, we demonstrate the application of our suggested methods. We will model sampling in the small statistical areas of the city of Tel-Aviv, Israel as considered in the recent Israeli census, and evaluate the performance of the different estimators. We will also introduce results under some perturbations of the model. The application involves estimation of certain population's proportions in small areas (Statistical Areas). The explanatory variables available when estimating the proportion $p_{i}$ in statistical area $i$, are 'Spatial' and 'Temporal', based on historical data, and data from neighboring statistical areas. We elaborate on comparing PEB procedures, versus the more recent NPEB procedure, suggested by Brown and Greenshtein 
(2009). In Section 4 we demonstrate the performance of our method on the baseball data studied by Brown (2009) and by Jiang and Zhang (2010).

Our ideas and techniques are meaningful in a genreral setup where $Y_{i} \sim F_{\mu_{i}}$, but will be presented for the case $F_{\mu_{i}} \equiv N\left(\mu_{i}, 1\right), i=1, \ldots, n$. In fact, as mentioned we will apply our method for estimating proportions in the setup where $Y_{i} \sim B\left(m_{i}, p_{i}\right)$, but applying an arcsin transformation will bring us to the normal setup.

\section{Collections of estimators induced by affine trans- formations}

The setup we consider is where we observe vectors $\boldsymbol{V}_{i}=\left(Y_{i}, X_{i 1}, \ldots, X_{i p}\right), i=1, \ldots, n$, where $Y_{i} \sim N\left(\mu_{i}, 1\right)$ are independent response variables, and $X_{i j}$ are explanatory variables independent of $Y_{i}, i=1, \ldots, n, j=1, \ldots, p$. Denote by $X_{n \times p}$ the matrix of the explanatory variables. Denote, $\boldsymbol{Y}^{\prime}=\left(Y_{1}, \ldots, Y_{n}\right)$. The goal is to find a 'good' estimator $\hat{\boldsymbol{\mu}}=\hat{\boldsymbol{\mu}}\left(\boldsymbol{V}_{1}, \ldots, \boldsymbol{V}_{n}\right)$, under the risk

$$
E\|\hat{\boldsymbol{\mu}}-\boldsymbol{\mu}\|_{2}^{2}
$$

In a nutshell the motivation and approach are as follows. Ideally it could be desired to approximate the Bayes procedure, assuming (at leat tactically) that $\left(\boldsymbol{V}_{i}, \mu_{i}\right), i=1, \ldots, n$, are independent random vectors sampled from an unknown distribution $\Gamma$ that belongs to a large non-parametric family of distributions $\mathcal{G}$. Then, the goal is to approximate the Bayes decision $\delta^{*}=\operatorname{argmin}_{\delta} E_{\Gamma}\left\|\delta\left(\boldsymbol{V}_{\boldsymbol{i}}\right)-\mu_{i}\right\|^{2}$ by $\hat{\delta}^{*}$, and let $\hat{\boldsymbol{\mu}}=\left(\hat{\delta}^{*}\left(\boldsymbol{V}_{\mathbf{1}}\right), \ldots, \hat{\delta}^{*}\left(\boldsymbol{V}_{\boldsymbol{n}}\right)\right)$. However, this goal may be too ambitious for $p+1$ dimensional observations $\boldsymbol{V}_{i}$ when $n$ is moderate due to the "curse of dimensionality". A possible approach, in the spirit of Lindley and Smith (1972), is to assume that $\Gamma$ belongs to a convenient parametric family, and this way the "curse of dimensionality" and other difficulties are circumvented. The approach of Fay and Herriot (1979) may also be interpreted this way. We, on the other hand, aim for the best permutational invariant estimator with respect to $Z_{1}, \ldots, Z_{n}$, where $Z_{i}$ are one dimensional random variables which are obtained by a suitable transformation of $\left(\boldsymbol{V}_{1}, \ldots, \boldsymbol{V}_{n}\right)$. This transformation is estimated from the data.

\section{1 preliminaries and definitions}

We start from a general point of view, where initially there are no covariates. We observe independent $Y_{i} \sim N\left(\mu_{i}, 1\right), i=1, \ldots, n$. Let $\{T\}$ be a collection of affine transformations $T(\boldsymbol{Y})=T_{A, B}(\boldsymbol{Y})=A \boldsymbol{Y}-\boldsymbol{B}$, where $A$ is an orthonormal matrix and $\boldsymbol{B}$ is a vector. Then $\boldsymbol{Z}=T(\boldsymbol{Y})$ is distributed as a multivariate normal with mean vector denoted $\boldsymbol{\nu}, \boldsymbol{\nu}=A \boldsymbol{\mu}-\boldsymbol{B}$, and covariance matrix the identity. Let $\Delta=\Delta(\boldsymbol{Y})$ be a fixed estimator of the vector $\boldsymbol{\mu}$, which is not invariant under the group of affine transformations, i.e., $\Delta(T(\boldsymbol{Y})) \neq T(\Delta(\boldsymbol{Y}))$. Then, the pair $\Delta$ and $\{T\}$ defines a (non-trivial) class of decision functions $\left\{\Delta_{T}\right\}, T \in\{T\}$,

$$
\Delta_{T}(\boldsymbol{Y})=T^{-1}(\Delta(T(\boldsymbol{Y})) .
$$


Let

$$
T^{o p t}=\underset{T \in\{T\}}{\operatorname{argmin}} E_{\boldsymbol{\mu}}\left\|\Delta_{T}(\boldsymbol{Y})-\boldsymbol{\mu}\right\|_{2}^{2} \equiv \underset{T \in\{T\}}{\operatorname{argmin}} R(T, \boldsymbol{\mu})
$$

here

$$
R(T, \boldsymbol{\mu})=E_{\boldsymbol{\mu}}\left\|\Delta_{T}(\boldsymbol{Y})-\boldsymbol{\mu}\right\|_{2}^{2} .
$$

Our goal is to approximate $T^{o p t}$, and then estimate $\boldsymbol{\mu}$ by an approximation of $\Delta_{T}$ opt $(\boldsymbol{Y})$.

For every $T \in\{T\}$, suppose we have a good estimator $\hat{R}(T, \boldsymbol{\mu})$ for $R(T, \boldsymbol{\mu})$. Let $\hat{T}=$ $\operatorname{argmin}_{T \in\{T\}} \hat{R}(T, \boldsymbol{\mu})$. The usual approach, which we will follow, is to use the estimator $\hat{\boldsymbol{\mu}}=$ $\Delta_{\hat{T}}(\boldsymbol{Y})$. When the class $\{T\}$ is not too large, we expect only a minor affect of over-fitting, i.e., $R(\hat{T}, \boldsymbol{\mu}) \approx R\left(T^{o p t}, \boldsymbol{\mu}\right)$.

Example 1 (Wavelet transfrom) The above formulation describes many standard techniques. In fact any harmonic analysis of the data that starts with transforming the data by a standard transformation (e.g., Fourier transform) follows this outline. A special case is when $T(\boldsymbol{Y})=A \boldsymbol{Y}$, where $A$ is the matrix which transforms $\boldsymbol{Y}$ to a certain wavelet representation, then, typically, the mean of the transformed vector is estimated and transformed back, see Donoho and Johnstone (1994). Suppose that, $\{T\}=\{A\}$ is a collection of matrices that correspond to a collection of wavelet bases/"dictionaries". The problem of finding the most appropriate basis/transformation, is related to that of basis-pursuit, see e.g., Chen, et.al. (2001). The permutational invariant and non-linear decision functions $\Delta$ in those studies is soft/hardthresolds, Lasso, etc. As mentioned, procedures of a special interest for us are parametric and non-parametric EB.

Example 2 (Regression) Suppose that in addition to $\boldsymbol{Y}$ there is a fixed (deterministic!) matrix $X \in R^{n \times p}$. Consider the class of transformations $T(\boldsymbol{Y})=\boldsymbol{Y}-\boldsymbol{B}, \boldsymbol{B} \in\{\boldsymbol{B}\}$, where $\{\boldsymbol{B}\}$ is the collection of all vectors of the form $\boldsymbol{B}=X \boldsymbol{\beta}, \boldsymbol{\beta} \in R^{p}$. Note, in particular, that our transformations are non-random.

Remark 1 The formulation for a random set $\{T\}$, which is independent of $\boldsymbol{Y}$ is just the same. In the last example when $X_{n \times p}$ is random, we condition on the explanatory variables and arrive to a conditional inference version of the developement in the sequel. From a Bayesian perspective, assuming a joint distribution $\Gamma$ as above, conditional independence of the random set $\{T\}$ and $\boldsymbol{Y}$, conditional on the covariates, follows when we assume that $\boldsymbol{Y}$ and $X_{n \times p}$ are independent conditional on $\boldsymbol{\mu}$. We will remark later on the case where the random set of transformations is 'weakly dependent' on $\boldsymbol{Y}$.

The following fact is useful. Let $\boldsymbol{Z}=T(\boldsymbol{Y})$. Then $Z_{i} \sim N\left(\nu_{i}, 1\right)$ where $\boldsymbol{\nu}=T(\boldsymbol{\mu})$, and

$$
R(T, \boldsymbol{\mu})=E_{\boldsymbol{\mu}}\left\|\Delta_{T}(\boldsymbol{Y})-\boldsymbol{\mu}\right\|_{2}^{2}=E_{\boldsymbol{\nu}}\|\Delta(\boldsymbol{Z})-\boldsymbol{\nu}\|_{2}^{2}=R(I, \boldsymbol{\nu}) .
$$

In the last equality $I$ represents the identity transformation. When there is no real danger of confusion, the dependence on $T$ is suppressed. We will use equation (1) later to establish an estimator $\hat{R}(T, \boldsymbol{\mu})$ for $R(T, \boldsymbol{\mu})$. 
The following general three steps method, for estimating $\boldsymbol{\mu}$, suggests itself.

Step I: For every $T$, estimate $R(T, \boldsymbol{\mu})$ by $\hat{R}(T, \boldsymbol{\mu})$.

Step II: Find $\hat{T}=\operatorname{argmin}_{T} \hat{R}(T, \boldsymbol{\mu})$.

Step III: Get the estimator: $\hat{\boldsymbol{\mu}}=\hat{T}^{-1}(\Delta(\hat{T}(\boldsymbol{Y}))) \equiv \Delta_{\hat{T}}(\boldsymbol{Y})$.

Note, $\hat{T}$ may depend on $\boldsymbol{Y}$.

We summarize. The idea in this subsection is that by an appropriate affine transformation, that may depend on explanatory variables, we will arrive to a problem which is 'easier' for the procedure $\Delta$ to handle. For example, by choosing an appropriate wavelet basis we will arrive to a sparse $\boldsymbol{\nu}$, which, roughly, is easier to handle/estimate the sparser it is. More generally, in a rough sense, good permutaion invariant procedures $\Delta$, "prefer" to estimate sparse vectors $\boldsymbol{\nu}$, hence transforming the original problem to a sparse problem is useful. Indeed accounting for the explanatory variables in a 'good' way, often brings us to a correponding sparse $\boldsymbol{\nu}$. Moreover, by accounting for explanatory variables in a good way through a suitable transformation, the transformed variables may become (nearly) exchangeable; whence, applying a permutation invariant procedure $\Delta$ on the transformed variables becomes natural and appealing.

\subsection{The case where $\Delta$ is parametric empirical Bayes and the Fay-Herriot procedure.}

The purpose of this subsection is to provide motivation to the nonparametric approach, and to give a unified treatment and presentation to the more classical Fay-Herriot approach and to the nonparametric approach. We study the case where $\Delta$ is a parametric empirical Bayes that corresponds to the prior $N\left(0, \tau^{2}\right)$, where $\tau^{2}$ is unknown. When $\tau^{2}$ is known the corresponding Bayes estimator for $\mu_{i}$ is $\hat{\mu}_{i}=\frac{\tau^{2}}{\tau^{2}+1} Y_{i}$, and its risk is $\frac{\tau^{2}}{\tau^{2}+1}$. When $\tau^{2}$ is unknown, we replace $\tau^{2}$ by its estimate. For our level of asymptotics all consistent estimators $\hat{\tau}^{2}$ induce equivalent estimators $\hat{\mu}_{i}=\frac{\hat{\tau}^{2}}{\hat{\tau}^{2}+1} Y_{i}$, and the corresponding estimators are asymptotically equivalent to James-Stein estimator up to $o(n)$, see Efron and Morris (1973). By working in this level of asymptotic, the considerations in this subsection are valid for a wide class of PEB procedures, corresponding to various consistent methods of estimating $\tau^{2}$, including the J-S procedure. In particular, the risk in estimating a (deterministic) vector $\boldsymbol{\mu}$ by PEB (or James-stein's) method equals:

$$
\frac{n\|\boldsymbol{\mu}\|_{2}^{2}}{\|\boldsymbol{\mu}\|_{2}^{2}+n}+o(n)
$$

We now examine our three steps estimation scheme, adapted for parametric Empirical Bayes (or, for a James-Stein estimator $\Delta$ ). Note that, for every $T$ and the corresponding $\boldsymbol{\nu}$ and $Z_{i}$ we have: $R(I, \nu)=\frac{n\|\boldsymbol{\nu}\|_{2}^{2}}{\|\boldsymbol{\nu}\|_{2}^{2}+n}+o(n)$. Hence a plausible estimator for $R(T, \boldsymbol{\mu})$ is

$$
\hat{R}(T, \boldsymbol{\mu})=\hat{R}(I, \boldsymbol{\nu})=\max \left\{0, \frac{n\left(\sum Z_{i}^{2}-n\right)}{\left(\sum Z_{i}^{2}-n\right)+n}\right\}=\max \left\{0, \frac{n\left(\sum Z_{i}^{2}-n\right)}{\sum Z_{i}^{2}}\right\}
$$

Our three steps scheme adapted for parametric empirical Bayes $\Delta$ is the following. 
Step I: For every $T$ estimate $R(T, \boldsymbol{\mu})$ by $(2)$.

Step II: Find $\hat{T}=\operatorname{argmin}_{T} \hat{R}(T, \boldsymbol{\mu})$.

Step III: Get the estimator: $\hat{\boldsymbol{\mu}}=\hat{T}^{-1}(\Delta(\hat{T}(\boldsymbol{Y}))) \equiv \Delta_{\hat{T}}(\boldsymbol{Y})$.

Remark 2 In the case where $\{T\}$ corresponds to $\left\{\boldsymbol{B}=\boldsymbol{X} \beta: \beta \in R^{p}\right\}$, the optimization step II is trivial. We want to minimize the residuals $\sum Z_{i}^{2}$. This is achieved for $\tilde{\boldsymbol{B}}$ which is the projection of $\boldsymbol{Y}$ on the span of the columns of $\boldsymbol{X}$, i.e., for $\hat{T}(Y)=\boldsymbol{Y}-X \hat{\boldsymbol{\beta}}$, where $\hat{\boldsymbol{\beta}}$ is the ordinary least squares estimator. Upon realizing the last fact, it is easy to see that our above suggested method is the method of Fay and Herriot.

\subsection{A nonparametric empirical Bayes $\Delta$}

The statements and development in this sub-section are for nonparametric empirical Bayes procedure $\Delta$, as in Brown Greenshtein (2009), see appendix. A recent study in which the NPEB procedure that was suggested by Jiang and Zhang (2009) is extended to handle covariates, is Jiang and Zhang (2010).

Let $Z_{i} \sim N\left(\nu_{i}, 1\right)$ be independent. Denote by $\mathcal{R}(\boldsymbol{\nu})$, the Bayes risk that corresponds to the prior which is defined by the empirical distribution of $\boldsymbol{\nu}$. Let $f_{\boldsymbol{\nu}}=\frac{1}{n} \sum \phi\left(z-\nu_{i}\right)$, where $\phi$ is the density of a standard normal distribution. Then

$$
\mathcal{R}(\boldsymbol{\nu})=1-\int \frac{\left(f_{\boldsymbol{\nu}}^{\prime}(z)\right)^{2}}{f_{\boldsymbol{\nu}}(z)} d z=1-E_{\boldsymbol{\nu}} \frac{\left(f_{\nu}^{\prime}(Z)\right)^{2}}{\left(f_{\boldsymbol{\nu}}(Z)\right)^{2}}
$$

see Bickel and Collins (1983).

The following theorem follows from Brown and Greenshtein (2009). It is stated for a triangular array set up, in order to cover situations of sparse $\boldsymbol{\nu} \equiv \boldsymbol{\nu}^{n}$. At stage $n, Y_{i} \sim$ $N\left(\mu_{i}^{n}, 1\right)$ are independent and for any corresponding sequence $T^{n}, T^{n} \in\left\{T^{n}\right\}, Z_{i} \sim N\left(\nu_{i}^{n}, 1\right)$ are independent, $i=1, \ldots, n$.

Assumption 1 For every $\alpha>0$ and every sequence $T^{n}$ and the corresponding $\boldsymbol{\nu}^{n}$ we have $\max _{i}\left(\nu_{i}^{n}\right)-\min _{i}\left(\nu_{i}^{n}\right)=o\left(n^{\alpha}\right)$.

Assumption 2 For some $\alpha_{0}>0, n^{\left(1-\alpha_{0}\right)} \mathcal{R}\left(\boldsymbol{\nu}^{n}\right) \rightarrow \infty$ for every $T^{n}$ and corresponding $\boldsymbol{\nu}^{n}$.

Theorem 1 Under Assumptions 1 and 2, for every sequence $T^{n}$,

$$
R\left(I, \boldsymbol{\nu}^{n}\right)=E_{\boldsymbol{\nu}^{n}}\left\|\Delta(\boldsymbol{Z})-\boldsymbol{\nu}^{n}\right\|_{2}^{2}=(1+o(1)) n \mathcal{R}\left(\boldsymbol{\nu}^{n}\right)
$$

As explained in the appendix, the procedure $\Delta$ in Brown and Greenshtein requires a bandwidth $h=h_{n}$, which approaches slowly to zero. The rate that implies the result in Theorem 1 is $h_{n} \sqrt{\log (n)} \rightarrow \infty$. 
Given $Y_{i} \sim N\left(\mu_{i}, 1\right)$, and a transformation $T, T \in\{T\}$. Let $Z_{i}$ be the $i^{\prime}$ th coordinate of $\boldsymbol{Z}=T(\boldsymbol{Y})$. The last theorem, and equations (1) and (3) suggest the following estimator $\hat{R}(T, \boldsymbol{\mu})$ for $R(T, \boldsymbol{\mu})$,

$$
\hat{R}(T, \boldsymbol{\mu})=n-\sum\left[\frac{\left(\hat{f}_{\nu}^{\prime}\left(Z_{i}\right)\right)}{\hat{f}_{\boldsymbol{\nu}}\left(Z_{i}\right)}\right]^{2},
$$

where the density $f_{\nu}$ and its derivative are estimated, for example, by appropriate kernel estimates.

Only step I of our general three steps procedure should be adapted, and replaced by:

Step I: For every $T$ and corresponding $\boldsymbol{\nu}=\boldsymbol{\nu}(T)$, estimate $R(T, \boldsymbol{\mu})$ by (5).

Remark 3 Step II could be computationally very complicated when the set $\{T\}$ is large. In the case where $\{T\}$ corresponds to $\left\{\boldsymbol{B}=\boldsymbol{X} \beta: \beta \in R^{p}\right\}$, a plausible choice, which is computationally convenient is to use the least-squares residuals for $\hat{T}(\boldsymbol{Y})$, as in the PEB case. This choice could be far from optimal as will be demonstrated in the following Examples 3 and 4 and in the simulations section.

Note, minimizing $R(I, \boldsymbol{\nu})$ with respect to $\boldsymbol{\nu}=\boldsymbol{\nu}(T)$ is equivalent to finding the "most favorable" prior, rather than the more conventional task of finding the least favorable prior.

The above method is reasonable when the class $\{T\}$ is not too large (in a VC dimension sense) and the overfit affect is not significant, otherwise regularization may be required. Those considerations are beyond the scope of our paper.

Choosing the least squares residuals, as mentioned in the remarks above, may be very inefficient, since it might cause "smoothing" of the empirical distribution and low values of $\left(f_{\tilde{\boldsymbol{\nu}}}^{\prime}\right)^{2}$, which by (3) implies high risk. This could be caused, e.g., by transforming a sparse structure into a non-sparse one, as in the following Example 3, or by transforming a structure with well separated groups into a mixed structure, as in the Example 4.

Example $3 Y_{i} \sim N(1,1), i=1, \ldots, 2 m, 2 m=n$. Suppose we have only one (useless) explanatory variable $X_{i}=1$ if $i \leq m$ and 0 otherwise. Projecting $\boldsymbol{Y}$ on $X$, we get $\tilde{\boldsymbol{B}} \approx$ $(1, \ldots, 1,0, \ldots, 0)^{\prime}$ and $\boldsymbol{\nu}=\boldsymbol{\mu}-\tilde{\boldsymbol{B}} \approx(0, \ldots, 0,-1, \ldots,-1)^{\prime}$, which is much worst for empirical Bayes estimation than the original $\boldsymbol{\mu}$ : It is easy to see that $n \mathcal{R}(\tilde{\boldsymbol{\nu}})=O(n)$, while $n \mathcal{R}(\boldsymbol{\mu})=0$. From Theorem 1 we conclude that as $n \rightarrow \infty$ the advantage of the latter (trivial) transformation compared to the least squares residuals in terms of the risk is o $(n)$ compared to $O(n)$.

Example 4 Let $Y_{i} \sim N\left(\mu_{i}, 1\right)$ are independent, where $\mu_{i}=\mu_{1}$ for $i=1, \ldots, m$ and $\mu_{i}=-\mu_{1}$ for $i=m+1, \ldots, 2 m=n$. Suppose $X_{i}=\left(\mu_{i}+W_{i}\right) \sim N\left(\mu_{i}, 1\right)$, independent of $Y_{i}, i=1, \ldots, n$. Let $\tilde{\boldsymbol{\nu}}=\boldsymbol{\mu}-\tilde{\boldsymbol{B}}$ where $\tilde{\boldsymbol{B}}$ is the projection of $Y$ on the (random) vector $\boldsymbol{X}=\left(X_{1}, \ldots, X_{n}\right)^{\prime}$. It easy to check that $\tilde{\nu}_{i} \rightarrow \mu_{i} /\left(\mu_{1}^{2}+1\right)-\mu_{1}^{2} W_{i} /\left(\mu_{1}^{2}+1\right)$ as $n \rightarrow \infty$. When $\mu_{1} \rightarrow \infty$, the empirical distribution of $\tilde{\boldsymbol{\nu}} \equiv \boldsymbol{\nu}^{n}$ converges to that of a standard normal. The corresponding Bayes risk $\mathcal{R}\left(\tilde{\boldsymbol{\nu}}^{n}\right)$ converges to 0.5. Obviously the Bayes risk that corresponds to the trivial transformation, for which $\boldsymbol{\nu}^{n}=\boldsymbol{\mu}^{n}$, converges to zero. 


\subsection{Optimality of NPEB $\Delta$.}

Until this point the treatment was for a concrete procedure $\Delta$ and a class $\{T\}$ of transformations. The purpose of this section is to advocate the choice of a non-parametric empirical Bayes $\Delta$, which is denoted $\Delta_{N P}$.

However, as noted, the optimization step (Step II) in the non-parametric approach may be computationally intensive, so such dominance result might not be enough to persuade that the non-parametric approach might be a good alternative to the parametric approach and to the Fay Herriot procedure. In Theorem 2 below we show that for every two sequences $\boldsymbol{\mu}^{n}$ and $T^{n}$, the sequence of estimators, that is obtained by coupling $T^{n}$ with $\Delta_{N P}$, asymptotically dominates the sequence which is obtained when coupling the same $T^{n}$ with any other sequence of permutation invariant procedures $\Delta^{n}$.

Given a procedure $\Delta$, a transformation $T$, and a mean vector $\boldsymbol{\mu}$, the corresponding risk is denoted for simplicity as $R_{\Delta}(T, \boldsymbol{\mu}) \equiv R(T, \boldsymbol{\mu})$ as before; for the case of nonparametric EB procedure $\Delta_{N P}$, the corresponding risk is denoted $R_{N P}(T, \boldsymbol{\mu})$. Similarly to the previous subsection our asymptotic analysis is of a triangular array setup.

Theorem 2 Let $\boldsymbol{\mu}^{n}, \Delta^{n}$ and $T^{n}$ be arbitrary sequences. Assume that for each $n$ the procedure $\Delta^{n}$ is simple symmetric. Further assume Assumptions 1,2. Then:

$$
\limsup \frac{R_{N P}\left(T^{n}, \boldsymbol{\mu}^{n}\right)}{R_{\Delta^{n}}\left(T^{n}, \boldsymbol{\mu}^{n}\right)} \leq 1
$$

Proof: Follows from Brown and Greenshtein (2009) and Theorem 1. Note that, the risk of the optimal simple symmetric procedure equals $n \mathcal{R}\left(\boldsymbol{\nu}^{n}\right)$.

Conjecture: It seems that in Theorem 2, the condition that $\Delta^{n}$ are simple symmetric for every $n$, may be replaced by the weaker condition, that $\Delta^{n}$ are permutation invariant for every $n$. This should follow by an equivalence result in the spirit of Greenshtein and Ritov (2009), though stronger. Note, the equivalence result in Greenshtein and Ritov (2009) would suffice under the assumption that $\max _{i}\left(\nu_{i}^{n}\right)-\min _{i}\left(\nu_{i}^{n}\right)=O(1)$; however, Assumption 1 allows a higher order.

\subsection{Remark}

The following remark is for the case in which we are mainly interested, where $\{T\}$ corresponds to $\{\boldsymbol{B}=X \beta\}$. Denote $\boldsymbol{B}=\left(B_{1}, \ldots, B_{n}\right)^{\prime}$. In the application we have in mind the set $\{T\}$ may be random since $X_{i j}$ could be random. When the random set of transformations is independent of $\boldsymbol{Y}$, our above treatment applies by conditioning on the explanatory variables. We will be interested in situations where the random set $\{T\}$ may depend on $\boldsymbol{Y}$, however we will require that $Y_{i}$ is independent of $X_{i 1}, \ldots, X_{i p}$ for each $i$. Then the conditional distribution of $Z_{i}$ conditional on $X_{i 1}, \ldots, X_{i p}$ is $N\left(\nu_{i}, 1\right)$, where $\left(\nu_{1}, \ldots, \nu_{n}\right)^{\prime}=\boldsymbol{\nu}=A \boldsymbol{\mu}-\boldsymbol{B}$ as before. When the dependence of $Y_{i}$ on $X_{j 1}, \ldots, X_{j p}, j \neq i$ is not too heavy, a natural goal is still to try to 
approximate the best decision function for estimating $\nu_{i}$ among the decision functions which are simple symmetric with respect to $Z_{1}, \ldots, Z_{n}$. The conditional marginal distribution of $Z_{i}$, $i=1, \ldots, n$ is still $N\left(\nu_{i}, 1\right)$ (i.e., the conditional distribution of $Z_{i}=Y_{i}-B_{i}$ conditional upon $\left.\left(X_{i 1}, \ldots, X_{i p}\right)\right)$; however, we may not treat them as independent observations. Thus, the rates of estimating $f_{\nu}$ and its derivative may become slower, and for heavy dependence, Theorems 1 and 2 might not hold. Similarly, rates of estimation of $\tau_{n}^{2}$, in order to apply the PEB procedure, could be slow. However, when the dependence is not "too heavy" we may expect Theorems 1 and 2 to hold under the assumption that $Y_{i}$ is independent of $X_{i_{1}}, \ldots, X_{i p}$ for each $i$.

\subsection{Intermidiate Discussion and Summary}

In this subsection we will summarize and compare the various approaches, elaborating on the approach of Fay and Herriot and of Jiang and Zhang, in addition to our suggested approach. We will take the liberty to follow those approaches just in "spirit" in this subsection. From a compound decision (non-Bayesian) perspective all the approaches assume that $\boldsymbol{Y}$ is a multivariate normal random vector with mean vector $\boldsymbol{\mu}$, i.e., $\boldsymbol{Y}=\boldsymbol{\mu}+\boldsymbol{\epsilon}$, where $\boldsymbol{\epsilon}$ is multivariate normal with mean zero. Given a matrix $\boldsymbol{X}$ of explanatory variables let $\boldsymbol{\xi}^{\prime}$ be the projection of $\boldsymbol{\mu}$ on the linear space spanned by the collumns of $\boldsymbol{X}$. Then we may write $\boldsymbol{\mu}=\boldsymbol{\xi}^{\prime}+\boldsymbol{\xi}$, where $\boldsymbol{\xi}^{\prime}$ is orthogonal to $\boldsymbol{\xi}$. Assume for convenience that $\boldsymbol{X}$ is non-singular, then there is a unique $\boldsymbol{\beta}$ that satisfy:

$$
\boldsymbol{Y}=\boldsymbol{X} \boldsymbol{\beta}+\boldsymbol{\xi}+\boldsymbol{\epsilon}
$$

Under a Bayesian formulation the vector $\boldsymbol{\xi}$ is consisted of i.i.d. sampled variables $\xi_{i} \sim G$, often $G$ is assumed normal. Under Fay-Herriot approach, which is especially appealing when $G$ is assumed normal, we estimate $\boldsymbol{\beta}$, using least square estimator $\hat{\boldsymbol{\beta}}$; then we estimate the mean $E(\boldsymbol{Y}-\boldsymbol{X} \hat{\boldsymbol{\beta}}) \approx \boldsymbol{\xi}$ of the 'nearly' multivariate normal vector $(\boldsymbol{Y}-\boldsymbol{X} \hat{\boldsymbol{\beta}})$ by a variant of the James-Stein estimator, finally we transform back to obtain an estimator for $\boldsymbol{\mu}$. The approach of Jiang and Zhang is similar, only they prefer to estimate the mean of $\boldsymbol{Y}-\boldsymbol{X} \hat{\boldsymbol{\beta}}$ by a nonparametric mle as in Jiand and Zhang (2009). The later method is is appropriate for a general distribution $G$ under a Bayesian approach, or for a general vector $\boldsymbol{\xi}$ under a compound decision approach. Our approach differ from that of Jiang and Zhang by suggesting to estimate the mean of the transformed vector by the NPEB estimator that was suggested by Brown and Greenshtein (2009), however this is not our main "message" since both procedures have similar performance. As demonstrated in Examples 3 and 4 if we use NPEB or a non parametric mle procedure $\Delta$, it is not clear that it is at all useful to transform the problem and estimate the mean of $\boldsymbol{Y}-\boldsymbol{X} \hat{\boldsymbol{\beta}}$; estimating the mean of $\boldsymbol{Y}-\boldsymbol{X} \tilde{\boldsymbol{\beta}}$, for $\tilde{\boldsymbol{\beta}} \neq \hat{\boldsymbol{\beta}}$ could be far better.

Finding the appropriate alternative $\tilde{\boldsymbol{\beta}}$, could be complicated especially computationally. However, there are examples and applications where we could find such a better transformation by intelligent guessing. A general scenario is the following. Suppose that we have a plausible linear model with a certain $\boldsymbol{\beta}_{0}$, which works fine for most cases, but does not work for a few outliers that behave differently than most cases. Applying a transformation with $\tilde{\boldsymbol{\beta}}=\boldsymbol{\beta}_{0}$, 
would bring us to a situation where the mean of $\boldsymbol{Y}-\boldsymbol{X} \tilde{\boldsymbol{\beta}}$ is a sparse vector with many "nearly" zero components and a few components that are very different than zero; such a sparse mean vector is often "easier to estimate" compared to estimation of the mean vector of $\boldsymbol{Y}-\boldsymbol{X} \hat{\boldsymbol{\beta}}$, which could be far from sparse due to "oversmoothing". In the example presented in Section 3 , of estimating the vector of current proportions of registered people in various areas, a useful explanatory variable is the corresponding vector of proportions from the previous year. A linear relation with $\beta_{0}=1$ is appropriate for most areas, excluding areas that went through a rapid development in the last year. Indeed in our study of the census example in Section 3, we tried in addition to $\hat{\boldsymbol{\beta}}$ a few more candidates that were chosen through an "intelligent guess", and not through a numerical search.

\section{Census Example}

\subsection{Preliminaries}

The city of Tel Aviv, Israel, is divided into 161 small areas called "statistical areas", each area belongs to a sub-quarter that includes about four additional statistical areas. The recent Israeli census was based on administrative records corrected by samples. Thus the proportion $p_{i}$ of people who are registered in area $i$ among those who live in area $i, i=1, \ldots 161$, were of interest. The estimated $p_{i}, i=1, \ldots, n$ are used in order to adjust the administrativeregistration counts and get population estimates for each area. In the following example we use the parametric bootstrap concept to evaluate the performance of various estimators. In our parametric bootstrap we use for the parameters $p_{1}, \ldots, p_{n}$, their values as estimated in the recent census (where about $20 \%$ of the population was sampled). The mean of $p_{i}, i=1, \ldots, 161$, is 0.75 and their standard deviation is 0.13 , their histogram is roughly bell shaped.

We will present a bootstrap study in which $p_{i}, i=1, \ldots, 161$ are estimated based on samples of size $m_{i}$ and corresponding simulated independent $\tilde{Y}_{i}, \tilde{Y}_{i} \sim B\left(m_{i}, p_{i}\right)$. Here $\tilde{Y}_{i}$ is the number of people in the sample from area $i$, which are registered to area $i$.

In addition we will simulate covariates in our parametric bootstrap. We will simulate temporal variables that correspond to historical data from each area $i$, and spatial covariates, that correspond to samples from the neighboring areas of each area $i$. In the following we will explore scenarios for the cases of: only temporal covariates, only spatial covariates, and both temporal and spatial covariates. We will compare the performance of PEB, NPEB and other methods. In all the analyzed situations, we will simulate binomial observations with sample size $m_{i} \equiv m$, for $m=25,50,100$.

In order to reduce this setup to the above normal case, we apply an arcsin transformation on our binomial observations $\tilde{Y}_{i}, i=1, \ldots, n$, as in Brown (2008). Specifically, let

$$
Y_{i}=\sqrt{4 m} \arcsin \left(\sqrt{\frac{\tilde{Y}_{i}+0.25}{m+0.5}}\right) .
$$

Then, $Y_{i}$ are distributed approximately as $N\left(\sqrt{4 m} \arcsin \left(\sqrt{p_{i}}\right), 1\right)$. We estimate $\mu_{i}=E\left(Y_{i}\right)$, 
by $\hat{\mu}_{i}, i=1, \ldots, n$, as explained in sub-sections 2.3 and 2.3 , and then let the estimate of $p_{i}$, $i=1, \ldots, 161$ equal,

$$
\hat{p}_{i}=\left(\sin \left(\frac{\hat{\mu}_{i}}{\sqrt{4 m}}\right)\right)^{2}
$$

Let $\boldsymbol{p}=\left(p_{1}, \ldots, p_{n}\right)$ and $\left.\hat{\boldsymbol{p}}=\left(\hat{p}_{1}, \ldots, \hat{p}_{n}\right)\right)$. We evaluate the performance of an estimator according to the risk

$$
E_{\boldsymbol{p}}\|\hat{\boldsymbol{p}}-\boldsymbol{p}\|_{2}^{2}
$$

The risk is approximated through 1000 simulations for each entry in the tables in the sequel. A different parametric EB approach for estimating proportions in small areas, that involves a logistic regression model, may be found in Farell, et.al.

\section{$3.2 \quad$ Temporal Covariates}

We introduce now simulated scenarios with only Temporal covariates. We think of a process where each year a sample of size $m$ is taken from each area. Suppose we use the records of the previous three years as covariates. Let $\tilde{T}_{i}$ be the number of people among the $3 m$ that were sampled in the previous three years from area $i$, which were registered to the area. Although $\tilde{T}_{i}$ might be better modeled as a binomial mixture, we will model $\tilde{T}_{i}$ as $B\left(3 m, p_{i t}\right)$ for simplicity. In order to (hopefully) have a linear relation between the response and explanatory variable, we define our temporal covariates as:

$$
T_{i}=\sqrt{4 m} \arcsin \left(\sqrt{\frac{\tilde{T}_{i}+0.25}{3 m+0.5}}\right) .
$$

Note, if there is hardly any change from the previous three years to the current year in area $i$, we will have $p_{i} \approx p_{i t}$ and $E\left(T_{i}\right) \approx E\left(Y_{i}\right)$.

In the following we will simulate two scenarios. One scenario is of no-change where $p_{i t}=p_{i}$, $i=1, \ldots, 161$. The other scenario is of a few abrupt changes; specifically, $p_{i}=p_{i t}, i=$ $17, \ldots, 161$, however $p_{i t}=0.3<p_{i}$ for $i=1, \ldots, 16$. Such abrupt changes could occur in areas that went in previous years through a lot of building, internal immigration and other changes.

Since the empirical distribution of $E\left(Y_{i}\right)$ is roughly bell-shaped it is expected that the PEB method will work well in the no-change scenario. Under the few abrupt changes, an advantage of the NPEB procedure will be observed.

As mentioned in Section 2, the optimization step of the NPEB procedure is difficult. We will try two candidate transformations $Y-B^{i}, i=1,2$, coupled with the NPEB, the corresponding methods are denoted NPEB1 and NPEB2. NPEB1 corresponds to the leastsquares/Fay-Herriot transformation, while NPEB2 corresponds to the transformation $Z_{i}=$ $Y_{i}-T_{i}$ (i.e., $\boldsymbol{B}^{2}=\left(T_{1}, \ldots, T_{n}\right)^{\prime}$ ). The later transformation, although still sub-optimal when coupled with a NPEB $\Delta$, could occasionally perform better than the former, as also indicated by Examples 3 and 4 . In addition to comparing the risks of the PEB and NPEBi, $i=1,2$ methods, we will also compare the the risk of the naive estimator, and of the regression estimator. The regression estimator estimates $\hat{\mu}_{i}$ through the least squares predictor (i.e., $\hat{\boldsymbol{\mu}}=X \hat{\beta}$ ), however 
Table 1:

\begin{tabular}{|l|rrrrr|}
\hline & Naive & Reg & NPEB1 & NPEB2 & PEB \\
\hline$m=25$ & 1.12 & 0.33 & 0.35 & 0.37 & 0.27 \\
$m=50$ & 0.56 & 0.17 & 0.18 & 0.18 & 0.14 \\
$m=100$ & 0.28 & 0.092 & 0.093 & 0.093 & 0.073 \\
& & & & & \\
\hline
\end{tabular}

Table 2:

\begin{tabular}{|l|rrrrr|}
\hline & Naive & Reg & NPEB1 & NPEB2 & PEB \\
\hline$m=25$ & 1.12 & 1.66 & 0.75 & 0.49 & 0.68 \\
$m=50$ & 0.56 & 1.64 & 0.46 & 0.22 & 0.42 \\
$m=100$ & 0.28 & 1.62 & 0.26 & 0.11 & 0.24 \\
& & & & & \\
\hline
\end{tabular}

it does not apply an additional PEB or NPEB stage. The Naive estimator simply estimates $p_{i}$ by the corresponding sample proportion.

The no-change scenario is presented in Table 1. Each entry is based on 1000 simulated realizations. Under no-change the temporal covariate is very helpful, and even the regressionestimate, i.e. least squares linear predictor is doing very well. Over all, the Naive estimator is the worst, NPEB1, NPEB2 and Regression are about the same, while the PEB is moderately better than the other methods.

Next we consider the scenario of a few abrupt changes. In this scenario the regression by itself is performing the worst, however an additional EB step is helpful. Here the NPEB2 procedure is the best, see Table 2 .

\subsection{Spatial Covariates}

In this section we simulate a scenario with spatial covariates. Tel-Aviv is divided into subquarters, where a few statistical areas define a sub-quarter. Each sub-quarter is defined by about 5 statistical areas. For every $i=1, \ldots, 161$, we define the neighborhood of area $i "$, as all the statistical areas other than area $i$, that belong to the same sub-quarter as area $i$. 
Table 3:

\begin{tabular}{|l|rrrrr|}
\hline & Naive & Reg & NPEB1 & NPEB2 & PEB \\
\hline$m=25$ & 1.12 & 1.41 & 0.72 & 0.75 & 0.64 \\
$m=50$ & 0.56 & 1.34 & 0.44 & 0.44 & 0.40 \\
$m=100$ & 0.28 & 1.31 & 0.26 & 0.28 & 0.23 \\
& & & & & \\
\hline
\end{tabular}

Based on the census we have good estimates for $p_{i s^{-}}$the proportion of people living in the neighborhood of area $i$, who are registered to their areas. Those estimates are treated as the "real" values in our simulations. The correlation between $p_{i}$ and $p_{i s}, i=1, \ldots, 161$ is 0.62 .

For simplicity we will assume that for each $i$, the size of the sample from the neighborhood of area $i$ is $4 m$. Let $\tilde{S}_{i}$ be the number of people sampled from the neighborhood of $i$, who are registered to their area. Although $\tilde{S}_{i}$ might be better modeled as a binomial mixture, we will model $\tilde{S}_{i}$ as $\tilde{S}_{i} \sim B\left(4 m, p_{i s}\right)$ for simplicity. As in the case of Temporal covariates we define the Spatial covariate for area $i$ as:

$$
S_{i}=\sqrt{4 m} \arcsin \left(\sqrt{\frac{\tilde{T}_{i}+0.25}{4 m+0.5}}\right) .
$$

As in the temporal case we will consider two NPEB estimates, corresponding to the projection/FayHerriot and to the $Z_{i}=Y_{i}-S_{i}$ transformations. The results of our simulations are summarized in Table 3. The advantage of the EB procedures is more noticeable for small $m=25$. The explanation is the following. Since the temporal covariate is not very strong, $\boldsymbol{\nu}$-the mean of the transformed variables is not too sparse. When $m$ is large, under the scale which is induced by the variance of $Z_{i}$, the points $\nu_{i}, i=1, \ldots, n$, may be viewed as isolated (i.e., extremely non sparse) and the smoothing of the EB is hardly effective. Hence the EB methods behave roughly like the Naive estimator.

One could wonder whether the spatial covariates are helpful at all, for the non parametric empirical Bayes, i.e, may be it is better not to transform the data at all and to apply $\Delta_{N P}$ on the original data taking $T=I$ and $\boldsymbol{\nu}=\boldsymbol{\mu}$. However this option is slightly worst than the above ones. The simulated risks that correspond to $m=25,50,100$ are $0.84,0.5$ and 0.28 .

\subsection{Spatial and Temporal Covariates.}

In this sub-section we study the performances of our estimators when both the temporal and spatial variables are introduced. As before we will apply the projection transformation for the NPEB estimator. However, we will also try the transformations $Z_{i}=Y_{i}-\left(\alpha S_{i}+(1-\alpha) T_{i}\right)$, 
Table 4:

\begin{tabular}{|l|rrrrrrr|}
\hline & Naive & Reg & NPEB1 & NPEB2 & NPEB3 & NPEB4 & PEB \\
\hline$m=25$ & 1.12 & 1.13 & 0.65 & 0.49 & 0.54 & 0.55 & 0.58 \\
$m=50$ & 0.56 & 1.06 & 0.4 & 0.22 & 0.28 & 0.38 & 0.37 \\
$m=100$ & 0.28 & 1.03 & 0.24 & 0.11 & 0.15 & 0.22 & 0.22 \\
& & & & & & & \\
\hline
\end{tabular}

for $\alpha=0,0.3,0.6$. The corresponding estimators are denoted: NPEB1 (for the projection), NPEB2, NPEB3 and NPEB4 correspondingly. For the temporal covariates we simulate the scenario of 16 abrupt changes, the spatial covariates are as before. As may be expected, since the spatial covariate is weak relative to the temporal, accounting for it causes extra unnecessary smoothing. For the non-parametric EB procedure, indeed NPEB2 that corresponds to $\alpha=0$ has the best performance, which is also the optimal among all the seven methods.

\section{Baseball Example}

In this section we will analyze the Baseball data set, originally analyzed by Brown (2009) and later by Jiang and Zhang (2010). Our analysis resambles that of Jiang and Zhang. The data is consisted of batting records of each Major league player in 2005. For each player $i$, denote by $N_{1 i}$ and $H_{1 i}$ the number of at bats and the number of hits he had in the first half of the season; similarly $N_{2 i}$ and $H_{2 i}$ are the corresponding quantities for the second half. In addition, for every player it is known whether he is a pitcher or a batter. For $j=1,2$ denote

$$
R_{j i}=\frac{H_{j i}}{N_{j i}}
$$

Note, in the example of the previous section the "number of trials" or "sample size" was denoted $m_{i}$, we use the notation $N_{j i}$ in order to keep notations close to those of Brown and of Jiang and Zhang.

Our purpose is to predict the value of $R_{2 i}$ for player $i$, based on the data from the first half.

A reasonable model for the data is that conditional on $N_{j i} i=1, \ldots, n, j=1,2, H_{j i} \sim$ $\operatorname{Bin}\left(N_{j i}, p_{i}\right)$, where $p_{i}$ is the (fixed in time) probability of a successful hit by player $i$. Thus a reasonable approach is to estimate $p_{i}$ by $\hat{p}_{i}$ and let our predictor for the value of $R_{2 i}$ be $\hat{R}_{2 i}=\hat{p}_{i}$, $i=1, \ldots, n$. So, similarly to our previous example we should estimate the proportions $p_{i}$.

Denote $S_{j}=\left\{i \mid N_{j i} \geq 11\right\}$. The estimation of $p_{i}$ will be done only for players $i$, such that 
$i \in S_{1}$. Validation of the prediction $\hat{p}_{i}$ will be made only for players $i$ such that $i \in S_{2}$. The size of $S_{1}$ is 567 , while the size of $S_{1} \cap S_{2}$ is 499 .

The criterion for the performance of a predictor $\hat{R}_{2 i}, i=1, \ldots, 499$, is based on the following estimator of $E \sum_{i \in S_{1} \cap S_{2}}\left(\hat{R}_{2 i}-R_{2 i}\right)^{2}$, denoted $T \hat{S} E_{R}$,

$$
T \hat{S} E_{R}=\sum_{i \in S_{1} \cap S_{2}}\left(\hat{R}_{2 i}-R_{2 i}\right)^{2}-R_{2 i}\left(1-R_{2 i}\right) / N_{2 i}
$$

As a benchmark for the performance of $\hat{\boldsymbol{R}}$ we take the performance of the naive estimator $\hat{R}_{2 i}=R_{1 i}$, specifically the value $T \hat{S} E_{0}=\sum_{i \in S_{1} \cap S_{2}}\left(R_{1 i}-R_{2 i}\right)^{2}-R_{2 i}\left(1-R_{2 i}\right) / N_{2 i}$. We will report the results of an estimator $\hat{R}_{2 i}, i=1, \ldots, 499$, through:

$$
T \hat{S} E_{R^{*}}=\frac{T \hat{S} E_{R}}{T \hat{S} E_{0}}
$$

\subsection{Covariates and transformations}

We consider a few "linear models" with the following covariates. One covariate for player $i$ is the number of trials $N_{1 i}$ he had. Note, $N_{1 i}$ corresponds to $m_{i}$ in the previous section. The additional covariate is indicator of the event that the player is not a pitcher.

The value of $N_{1 i}$ is potentially a useful covariate, since that a high value of $N_{1 i}$ indicates that the coach percieve player $i$ as a good batter.

Our response variable for player $i$ is:

$$
Y_{i}=\sqrt{4 * N_{1 i}} \arcsin \left(\sqrt{\frac{H_{1 i}+0.25}{N_{1 i}+0.5}}\right)
$$

Note, in the above we transformed the variables and obtained a homoscedastic model. Both Brown (2010) and JIang and Zhang (2010), worked in a heteroscedastic setup with the variables $Y_{i}^{*}$, where $Y_{i}^{*}=\arcsin \left(\sqrt{\frac{H_{1 i}+0.25}{N_{1 i}+0.5}}\right)$.

Jiand and Zhang (2010) considered the following linear models all include intercept. Using their notations we denote the covariate $N_{1 i}$, the number of At Bat of player $i$, by $\mathrm{AB}$. The models they considered with respect to $y_{i}^{*}$ are: i) AB ii) Pitcher iii) Pitcher $+\mathrm{AB}$ iv) Pitcher $+\mathrm{AB}+$ Pitcher*AB. In the above we used the standard notations, where the last model includes interaction of the variables $\mathrm{AB}$ and Pitcher. The corresponding models in terms of our response variable $Y_{i}$ are: i) $A B^{0.5}+A B^{1.5}$ ii) $A B^{0.5}+A B^{0.5} *$ Pitcher iii) $A B^{0.5}+A B^{0.5} *$ Pitcher $+A B^{1.5}$ iv) $A B^{0.5}+A B^{0.5} *$ Pitcher $+A B^{1.5}+A B^{1.5} *$ Pitcher. Note, the intercept variable is transformed to $A B^{0.5}$ when modelling with respect to $Y_{i}$.

Again!, since we do not have a special insight/ "intelligent guess" or an efficient computation and searching algorithm, in each case we chose the transformation that corresponds to the least squares $\hat{\boldsymbol{\beta}}$. 


\subsection{Numerical Study.}

We will report the results of the above estimator on the baseball data. The results are slightly inferior compared to those achieved by the method WGMLEB of Jiang and Zhang, yet the computation of our estimator seems significantly easier.

We will consider three "models". The explanatory variable $N_{1 i}$ of the number At Bat is denoted by AB in Jiang and Zhang. The models are PITCHER, AB, and PITCHER+AB. We report on the performane of the following three methods. The variant of NPEB described above, Strict Regression without a second stage of NPEB, and WGMLEB (weighted general maximum likelihood empirical Bayes). The WGMLEB method had the best performance in the study of Jiang and Zhang (2010).

Model i)

The corresponding $T \hat{S} E_{R}^{*}$ for WGMLEB, NPEB and REGRESSION are: $0.291,0.353,0.526$

Model: ii)

The corresponding $T \hat{S} E_{R}^{*}$ for WGMLEB, NPEB and REGRESSION are: $0.204,0.234,0.343$

Model iii)

The corresponding $T \hat{S} E_{R}^{*}$ for WGMLEB, NPEB and REGRESSION are: $0.175,0.186,0.214$

Model iv)

The corresponding $T \hat{S} E_{R}^{*}$ for WGMLEB, NPEB and REGRESSION are: $0.167,0.162,0.200$

In the analysis made by Brown (2009), the covariate AB was used implicitly, through the estimation of the density $f$ and its derivative ( see appendix) in his variant of NPEB. The density at a point $y_{i}$ was estimated based on observations $y_{k}$, with $N_{1 k}$ "close" to $N_{1 i}$. This implicit use yielded $T \hat{S} E_{R}^{*}=0.509$ of the corresponding NPEB. The more direct approach we took seems beneficial.

\section{Discussion and Summary}

In this paper we studied the problem of extending Empirical Bayes methods so they may be naturally applied in situations where there are explanatory variables.

We suggested a general perspective in which the method of Fay and Herriot and our newly proposed "NPEB with covariates" method are special cases. We demonstrated through Examples 3,4, and more generally through Theorem 2 that asymptotically the NPEB method is advantageous over the method of Fay and Herriot and over a larger class of other methods. We demonstrated it also in a real data example, where it may be seen that our newly proposed method could be occasionally a good alternative to the method of Fay and Herriot in practical situations.

Some computational aspects of our newly proposed method should be further studied, but we showed that even sub-optimal (simpler to compute) versions of the method are advantageous. 


\section{Appendix}

NPEB procedure. We will follow the approach of Brown and Greenshtein (2009), see that paper for further details.

Assume $Z_{i} \sim N\left(\nu_{i}, \sigma^{2}\right), i=1, \ldots, n$, where $\nu_{i} \sim G$.

Let

$$
f(z)=\int \frac{1}{\sigma} \varphi\left(\frac{z-\nu}{\sigma}\right) d G(\nu) .
$$

It may be shown that the normal Bayes procedure denoted $\delta_{N}^{G}$, satisfies:

$$
\delta_{N}^{G}(z)=z+\sigma^{2} \frac{f^{\prime}(z)}{f(z)} .
$$

The procedure studied in Brown and Greenshtein (2009), involves an estimation of $\delta_{N}^{G}$, by replacing $f$ and $f^{\prime}$ in (10) by their kernel estimators which are derived through a normal kernel with bandwidth $h$. Denote the kernel estimates by $\hat{f}_{h}$ and $\hat{f}_{h}^{\prime}$ we obtain the decision function, $\left(Z_{1}, \ldots, Z_{n}\right) \times z \mapsto R$ :

$$
\delta_{N, h}(z)=z+\sigma^{2} \frac{\hat{f}_{h}^{\prime}(z)}{\hat{f}_{h}(z)} .
$$

A suitable (straightforward) truncation is applied when estimating the corresponding mean of points $Z_{i}$ for which $\hat{f}\left(Z_{i}\right)$ is too close to zero and consequently $\left|\delta_{N, h}\left(Z_{i}\right)-Z_{i}\right|>2 \log (n)$. We did not apply such truncation in our simulations in this paper. The default choice for the bandwidth $h \equiv h_{n}$, suggested by Brown and Greenshtein is $1 / \sqrt{\log (n)}$. See also, a cross-validation method for choosing $h$, suggested by Brown, et.al., (2010), together with some suggested improvements of the above procedure. In our numerical study for both examples, we chose $h=0.4$. The procedure is not too sensitive to the choice of $h$.

\section{References}

Bickel, P. J. and Collins, J.R. (1983). Minimizing Fisher information over mixtures of distributions. Sankhya Vol 45, No. 1, p 1-19.

Brown, L. D. (2008). In-Season Prediction of Bating Averages: A field test of Simple Empirical Bayes and Bayes Methodologies. Ann. of App. Stat. 2 113-152.

Brown, L.D. and Greenshtein, E. (2009). Non parametric empirical Bayes and compound decision approaches to estimation of high dimensional vector of normal means. Ann. Stat. 37, No 4, 1685-1704.

Brown, L.D, Greenshtein, E. and Ritov, Y. (2010). The Poisson compound decision problem revisited. Manuscript.

Chen, S.S, Donoho, D.L., Saunders, M. A (2001). Atomic decomposition by basis pursuit. SIAM Rev. Vol 43, Issue 1, 129-159. 
Copas, J.B. (1969). Compound decisions and empirical Bayes (with discussion). JRSSB 31 397-425.

Donoho, D.L. and Johnstone, I.M (1994). Ideal spatial adaptation by wavelet shrinkage. Biometrika 81 No. 3, 425-455.

Efron, B. and Morris, C. (1973). Stein's estimation rule and its competitors- an Empirical Bayes approach. JASA 68 117-130.

Efron, B. (2003). Robbins, Empirical Bayes, and Microarrays (invited paper). Ann.Stat 31, No. 2, 364-378.

Fay, R.E. and Herriot, R. (1979). Estimates of income for small places: An application of James-Stein procedure to census data. JASA, 74, No. 366, 269-277.

Farrell, P.J., MacGibbon, B., Tomberlin, T.J. (1997). Empirical Bayes estimators of small area proportions in multistage designs. Stat. Sinica 7 1065-1083.

Greenshtein, E., Park, J., Ritov, Y. (2008). Estimating the mean of high valued observations in high dimensions. Journal of Stat. theory and pract. 2 No.3, 407-418.

Greenshtein, E. and Ritov, Y. (2009). Asymptotic efficiency of simple decisions for the compound decision problem. The 3'rd Lehmann Symposium. IMS Lecture Notes Monograph Series, J.Rojo, editor.

Jiang, W. and Zhang, C.-H. (2009). General maximum likelihood empirical Bayes estimation of normal means. Ann. Stat. 37, No 4, 1647-1684.

Jiang, W. and Zhang, C.-H. (2010). Empirical Bayes in-season prediction of baseball batting averages. Borrowing Strength: Theory Powering Applications-A festschrift for L.D. Brown. J.O. Berger, T.T. Cai I.M. Johnstone, eds. IMS collections Vol 6, 263-273.

Lindley, D.V. and Smith, A.F.M. (1972). Bayes estimates for the linear model. JRSSB 34, No.1, 1-41.

Rao, J.N.K. (2003). Small area estimation. Wiley \& Sons, New Jersey.

Robbins, H. (1951). Asymptotically subminimax solutions of compound decision problems. Proc. Second Berkeley Symp. 131-148.

Robbins, H. (1955). An Empirical Bayes approach to statistics. Proc. Third Berkeley Symp. $157-164$.

Robbins, H. (1964). The empirical Bayes approach to statistical decision problems. Ann.Math.Stat. 35, $1-20$. 
Zhang, C.-H.(2003). Compound decision theory and empirical Bayes methods.(invited paper). Ann. Stat. 31 379-390.

Central Bureau of Statistics, Jerusalem, Israel

E-mail: noamc@cbs.gov.il

Central Bureau of Statistics, Jerusalem, Israel

E-mail: eitan.greenshtein@gmail.com

The Hebrew University of Jerusalem, Israel

E-mail: yaacov.ritov@gmail.com 